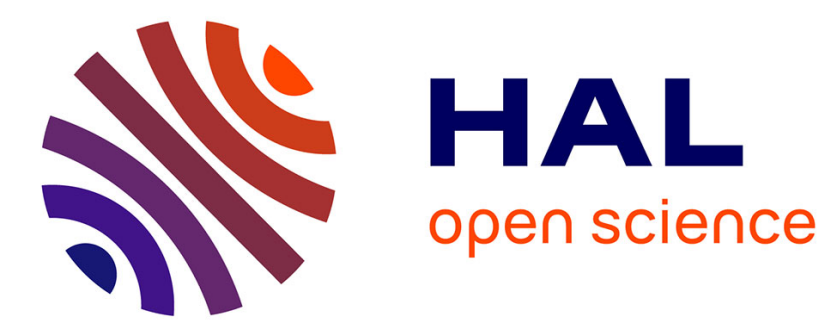

\title{
Theory of multiple ionization of xenon under strong XUV radiation and the role of the giant resonance
} Resonance P Lambropoulos, K G Papamihail, P Decleva

\section{To cite this version:}

Resonance P Lambropoulos, K G Papamihail, P Decleva. Theory of multiple ionization of xenon under strong XUV radiation and the role of the giant resonance. Journal of Physics B: Atomic, Molecular and Optical Physics, 2011, 44 (17), pp.175402. 10.1088/0953-4075/44/17/175402 . hal-00651654

\section{HAL Id: hal-00651654 \\ https://hal.science/hal-00651654}

Submitted on 14 Dec 2011

HAL is a multi-disciplinary open access archive for the deposit and dissemination of scientific research documents, whether they are published or not. The documents may come from teaching and research institutions in France or abroad, or from public or private research centers.
L'archive ouverte pluridisciplinaire HAL, est destinée au dépôt et à la diffusion de documents scientifiques de niveau recherche, publiés ou non, émanant des établissements d'enseignement et de recherche français ou étrangers, des laboratoires publics ou privés. 


\title{
Theory of Multiple Ionization of Xenon under strong XUV radiation and the role of the Giant Resonance.
}

\author{
P.Lambropoulos ${ }^{1,2}$, K.G.Papamihail ${ }^{1,2}$ and P.Decleva ${ }^{3}$ \\ ${ }^{1}$ Institute of Electronic Structure and Laser, FORTH, P. O. BOX 1527, GR-71110, \\ Heraklion, Greece \\ 2 Department of Physics, University of Crete, P. O. BOX 2208, GR-71003, Heraklion, \\ Crete, Greece \\ ${ }^{3}$ Dipartimento di Scienze Chimiche, Universita di Trieste, Via L. Giorgieri 1, I 34127 \\ Trieste, Italy \\ E-mail: labro@iesl.forth.gr
}

\begin{abstract}
.
We examine the conditions under which multiple ionization of Xenon, under strong radiation of photon energy $93 \mathrm{eV}$ and pulse duration $10 \mathrm{fs}$, leading up to $\mathrm{Xe}^{21+}$ can be observed. Through a set of rate equations, we show that the dominant sequential channels are sufficient for and consistent with the observed ionic species. Addressing the possible role of the giant resonance in this context, we evaluate the evolution of the neutral which is responsible for that resonance, examining its depletion during the pulse. We show that, owing to the large value of the cross section and in combination with the pulse duration, as the intensity rises towards the peak value, the neutral is depleted very early, at lower intensities, thus precluding its exposure to the higher peak intensities above $10^{15} \mathrm{~W} / \mathrm{cm}^{2}$. But we do point out that under much shorter pulse duration, direct many-electron multiple ejection may be a possibility, but not under the presently available conditions of intensity and pulse duration at the FEL (Free Electron Laser) facilities.
\end{abstract}

PACS numbers: $32.80 . \mathrm{Rm}, 32.80 . \mathrm{Fb}, 30.80 . \mathrm{Hd}, 42.50 . \mathrm{Hz}$ 


\section{INTRODUCTION}

Photo-absorption at short wavelength radiation, by which we mean the XUV and X-ray range, traditionally studied at synchrotron facilities, owing to limitations in the intensity of even the most advanced sources of that type, had been confined to singlephoton processes. This has changed with the recent appearance of new radiation sources at that wavelength range, such as the accelerator-based Free Electron Lasers (FEL) [1, which have made possible the observation of photo-absorption driven by sub-picosecond pulses of intense radiation, leading inevitably to multiphoton (non-linear) processes. A number of experimental data, as well as related theoretical studies, especially in the rare gases, have already been published [2, 3, 4. The first issue arising in this new context is whether the coupling to the radiation can still be described by LOPT (Lowest (non-vanishing) Order of Perturbation Theory). As long as the ponderomotive energy $U_{p}$ (cycle averaged kinetic energy of a free or loosely bound electron, in the field) is significantly smaller than the photon energy, LOPT is valid. To provide a point of calibration, note that for infrared radiation of photon energy $1 \mathrm{eV}$ and intensity $10^{13} \mathrm{~W} / \mathrm{cm}^{2}$, $U_{p}=1.43 \mathrm{eV}$, while it scales proportionally to the radiation intensity and inversely proportionally to the square of the photon energy. Then it follows that, for photon energies around $100 \mathrm{eV}$, the range of interest in this paper, LOPT is valid, as long as the intensity is below $10^{19} \mathrm{~W} / \mathrm{cm}^{2}$, which is three orders of magnitude larger than the intensities employed in our calculations and related experiments. In addition, for the notion of the cross section to be applicable, the pulse duration should be at least 10 cycles of the field; a condition amply satisfied, at this photon energy, even for a pulse as short as 1 fs.

Due to the large peak intensity (up to about $10^{16} \mathrm{~W} / \mathrm{cm}^{2}$ ), however, multiple ionization leading to the appearance of ionic species up to $\mathrm{Xe}^{21+}$ have been reported in TOF (Time of Flight) data [3, 4, The first question that multiple ionization raises is whether sequential processes, leading to successive ionic species, represent the dominant mechanism, or whether the simultaneous ejection of several electrons is also a major contributing process and if so, what would the underlying mechanism be? This is of particular relevance to Xe, known to exhibit a so-called giant resonance at photon energy $100 \mathrm{eV}$, which involves a single-photon ejection from the 4d shell. An overall 
theoretical interpretation [5], in so far as the dependence of the ionic species on laser intensity is concerned, up to about $\mathrm{Xe}^{15+}$, has shown that the underlying mechanism is indeed compatible with sequential ionization; beginning with the single-photon ejection of a $4 \mathrm{~d}$ electron and the subsequent Auger process producing (with known branching ratios) one or two more electrons [6]. As a consequence of the relevant branching ratios, the most abundant species, throughout the intensity range covered in the experiments [2, 3], is $\mathrm{Xe}^{2+}$, followed by $\mathrm{Xe}^{3+}$.

Although the photon energies of the two sets of data, namely $93 \mathrm{eV}$ [2] and $90.5 \mathrm{eV}$ [3], do not fall on the peak of the giant resonance, they are well within its broad width of about $30 \mathrm{eV}$, which implies relatively large cross section (about $22 \mathrm{Mb}$ ) very close to the peak value. This has prompted the speculation, albeit in rather vague terms, that the proximity to the giant resonance, in the presence of high intensity, may somehow play a role in the creation of multiply ionized species; other than producing $\mathrm{Xe}^{2+}$ and $\mathrm{Xe}^{3+}$, which initiates the sequential route to the higher ions. Specifically, as stated in [3], under "high irradiance" i.e. $10^{16} \mathrm{~W} / \mathrm{cm}^{2}$, "due to the higher amplitudes, the collective oscillations within the $4 \mathrm{~d}$ shell may lead to the emission of more than one $4 \mathrm{~d}$ electron, up to all ten, more or less simultaneously and coherently". Clearly, for this scenario to be of any relevance, it is necessary for the neutral to be exposed to that "high irradiance". Given that, at these photon energies, the creation of up to $\mathrm{Xe}^{7+}$ is due to a sequence of single-photon absorptions, the issue is amenable to detailed calculation, which is the chief objective of this work.

This type of single-photon resonance has been studied, theoretically, as well as experimentally, quite extensively over the last few decades [6, 7, 8, 9]. The magnitude of this feature is unusually large in Xenon, due to the double well shape of the potential and the strong localization of the continuum $\mathrm{f}$-wave in the inner well at the resonance energy [7, 10, 11]. It appears also in some of the ionic species of Xenon, as well as in the neighboring elements, gradually disappearing in the higher ionization stages, as the resonance is drawn below threshold and is transferred to the bound $4 \mathrm{f}$ state [8, 9]. Although terminology may vary, it is well known that this resonance is essentially due to the $\mathrm{d} \rightarrow \mathrm{f}$ coupling matrix element and the overall behavior can be obtained even in a single-particle calculation [7, 12]. Accurate reproduction of the experimental cross section and its dependence on photon energy does of course require a sophisticated ap- 
proach, such as RPA (Random Phase Approximation) [13] or Density Functional [14, 15] which takes into account the correlation in this multielectron system. It must be emphasized at the outset that this giant resonance represents a photo-absorption process within lowest order perturbation theory (LOPT), as far as the coupling of the electrons to the radiation is concerned; which means that it is described by a transition probability per unit time (Fermi's golden rule), expressed in terms of a cross section. The intra-atomic couplings between the electrons are of course non-perturbative, as already mentioned above. And although the term collective is occasionally used in this context, it should be kept in mind that it is only one electron that is ejected by the photon. The Auger events that follow, leading to $\mathrm{Xe}^{2+}$ and $\mathrm{Xe}^{3+}$, represent intra-atomic processes which occur even far away from the resonance.

Our main objectives in this paper are: (a) To provide an extension and more detailed exposition of our earlier work [5], including now ionic species up to $\mathrm{Xe}^{21+}$, thus confirming that the sequential channels alone account for the appearance of species as high as $\mathrm{Xe}^{21+}$. (b) To present a quantitative assessment of the role of the giant resonance. (c) To connect the underlying physics to more recent data, pertaining to the validity of LOPT. (d) To discuss the notion of direct multielectron ejection, a process that may turn out to be observable in future experiments, if sub-fs pulses become available in this wavelength range.

A few comments on terminology may be helpful here, before entering the technical aspects of the work. What we mean by sequential ionization is the chain of processes in which the absorption of one or more photons, depending on the ionization potentials, leads to successive ionic species (see Appendix). It is understood that the time scales are such that the ion produced relaxes to its ground state, before the next step in the sequence. This does not preclude the possibility of an ion left in an excited state, from which the next absorption event can occur. Whether this is a significant contribution will depend on the specific situation, i.e. atomic species, photon energy, pulse duration and intensity, as well as on how detailed the experimental data are. Be that as it may, in our equations below, it is assumed that the ions relax to the ground state, before the next event and that this relaxation includes the possible Auger process, as in the case with neutral Xenon. In strong-field radiation-atom interactions, at long wavelength (infrared), the term non-sequential is used to denote a process in which, the first (valence) 
electron pulled by the field from the atom, is set into oscillation acquiring the ponderomotive energy and upon returning to the atom, ejects another electron via a collision. That is why this process is referred to as recollision. Obvioulsy, for this to be possible, the $U_{p}$ of the returning electron must be larger than the binding energy of the valence electron. This type of process is out of the question, in our context, because as already mentioned, the ponderomotive energy is too small to eject even a valence electron. Obviously, it is even smaller compared to the binding energy of a sub-valence electron. What is in principle possible is the direct multiphoton ejection of two or more electrons, by means of multiphoton absorption of the necessary number of photons, which has nothing to do with recollision. That is why the proper term is direct multielectron ejection. The case of 2-photon direct two electron ejection in Helium, first proposed in [16], is a problem that has already received considerable attention [17, 18, 19]. Its generalization to several electron multiphoton direct ejection was introduced very recently in [20], while its possible relevance to this case is presented later on, in section V.

\section{THEORETICAL FRAMEWORK AND MODELING.}

In an experiment under a pulsed source such as the FEL, the atoms are exposed to radiation that rises from zero to the peak value and then falls off to zero, within tens of femtoseconds. Specifically in [3], the full temporal width of the pulse at half maximum (FWHM) was about $10 \mathrm{fs}$, which is by far sufficiently long for the notion of the cross section to be applicable. Since the conditions of the radiation-atom interaction in that experiment were well within the validity of LOPT, the evolution of the ionic species during the pulse is describable in terms of a set of differential equations, referred to as rate or kinetic equations. Such rate equations are perfectly adequate for the interpretation of experiments in which only total ionic yields are measured. When more detailed data, such as photoelectron energy spectra and angular distributions are measured, a more detailed description in terms of density matrix equations may be required. The input to these rate equations is the temporal behavior of the pulse - a Gaussian being sufficient for our purposes - as well as the relevant cross sections. As already mentioned above, the sequence from $\mathrm{Xe}$ to $\mathrm{Xe}^{7+}$, via the intermediate ionic stages $\mathrm{Xe}^{+}, \mathrm{Xe}^{2+}, \ldots$, 

these processes exist in the literature [6, 8, 9, 10, having been obtained through ab initio calculations, as well as measured in related experiments. The sequence beyond $\mathrm{Xe}^{7+}$ involves 2-photon, 3-photon, etc. up to 7-photon absorption; the latter in the steps leading from $\mathrm{Xe}^{19+}$ to $\mathrm{Xe}^{21+}$. Cross sections for these multiphoton processes can be obtained through an approach of scaling [21] which has been tested repeatedly over the last 25 years (including successful comparison to experimental data) and was employed in [5]. The complete list of the differential equations as well as the relevant cross sections, are given in the Appendix, while a schematic outline of the channels involved in the process is shown in Fig. 1]

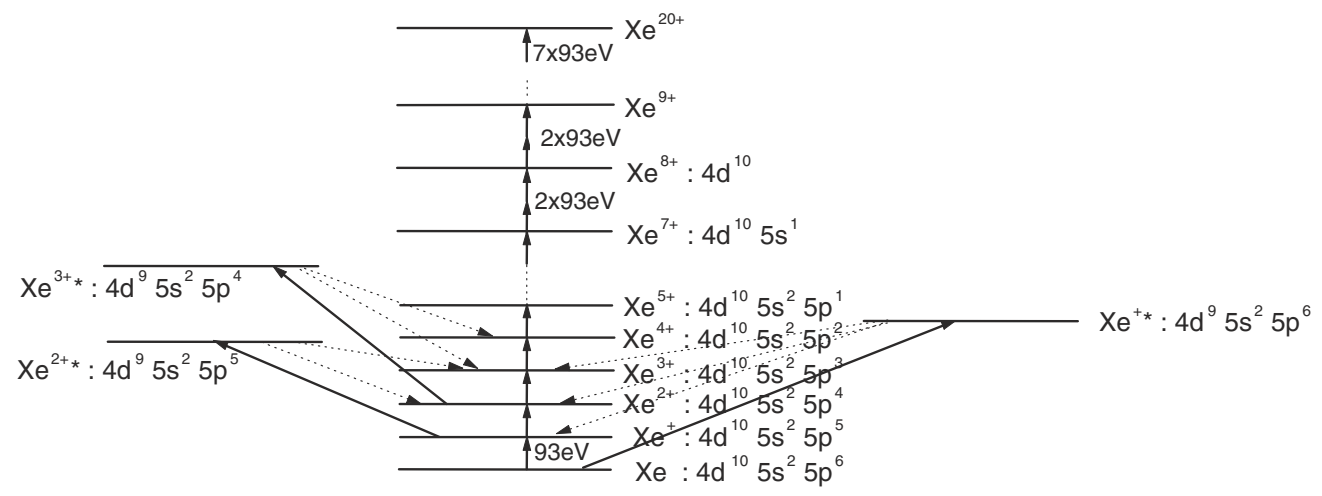

Figure 1. Schematic diagram of the main channels leading to sequential multiple ionization of Xe under radiation of photon energy $93 \mathrm{eV}$.

A pivotal issue in our present inquiry is the evolution of the lower ionic species and in particular neutral Xenon, during the pulse. That is because, if we are to speculate on the possibility that, owing to the giant resonance, neutral Xenon when exposed to the higher intensities reached in the experiment may exhibit unusual behavior, we need to ascertain that it will not be depleted during the rise of the pulse. Even if one were to be skeptical about the level of accuracy of the multiphoton cross sections entering the sequence of processes beyond $\mathrm{Xe}^{7+}$, they are of no consequence as far as the evolution of the neutral is concerned (see also relevant discussion in the Appendix). It should be obvious, but it bears emphasizing, that what happens to the neutral does affect the 
yield of the higher species, but not the other way around. Actually, mere inspection of the experimental ionic yields (Fig.4 in [2]) suggests that, at the intensity at which $\mathrm{Xe}^{8+}$ begins appearing in the data (around $8 \times 10^{13} \mathrm{~W} / \mathrm{cm}^{2}$ ), the neutral must have been depleted having given rise to the ionic species. And if we are to speculate about the possibility of all ten $4 \mathrm{~d}$ electrons being ejected "simultaneously", we should examine in particular the intensity at which $\mathrm{Xe}^{10+}$ begins appearing. We should at the same time examine whether the neutral, with its ten $4 \mathrm{~d}$ electrons, is still intact, when that intensity is reached. Again, on the basis of the experimental data (Fig.4 of [2]; where unfortunately the yield of $\mathrm{Xe}^{10+}$ is not shown), we see that the yields of species like $\mathrm{Xe}^{9+}$ and $\mathrm{Xe}^{11+}$, even at the highest peak intensity in the experiment $10^{16} \mathrm{~W} / \mathrm{cm}^{2}$, are two orders of magnitude below the yields of species like $\mathrm{Xe}^{2+}, \mathrm{Xe}^{3+}$ and $\mathrm{Xe}^{4+}$. Thus it is evident that, even at the qualitative level, the data themselves suggest that up to $\mathrm{Xe}^{7+}$ it is the single-photon processes that control the chain of events leading to the higher ions; simply because, if some 10-electron process played a significant role, one would expect a surge of $\mathrm{Xe}^{10+}$ quite early in the pulse, while the neutral is still available. And here is where the crucial dilemma has already surfaced. Early in the rise of the pulse, the neutral is available, but the intensity is low. Later, as the intensity approaches its peak, the neutral will have been depleted. But the experimental data alone cannot provide a definitive answer, as they do not contain information on the population of the neutral. Moreover, measured ionic yields, as shown in Fig.4 of [2], come not only from the focal region, where the highest peak intensity is reached, but also from an expanded peripheral volume of lower intensities. It is therefore theory that can provide the missing link, by examining the population of the neutral during the pulse in the focal region.

Proceeding now beyond the qualitative assessment, we consider the sequential channels, depicted schematically in Fig. 1. Using an expanded version of the rate equations of [5] (23 differential equations, shown in the Appendix) to calculate the ionic yields, as a function of peak intensity, for a pulse duration of $10 \mathrm{fs}$, including the population of the neutral, we obtain the result shown in Fig. 2. We have, on purpose, left out the spatial integration over the interaction volume [5], in order to exhibit the evolution (the appearance and disappearance) of the ionic species as a function of the laser peak intensity, at the focal region where the intensity is maximum. The effect of the spatial 
Theory of Multiple Ionization of Xenon under strong XUV radiation and the role of the Giant Resonance.8

integration (essentially an instrumental, albeit unavoidable effect) is well known and has, in the context of this problem, been included in [5]. In any case, as already noted above, the signal produced in the peripheral (outside the focal region) interaction volume is due to lower intensities.

Examining now the population of the neutral in Fig. 2. first note that it is practically gone (reduced by 5 orders of magnitude from its initial value of 1 ) at a peak intensity of about $6 \times 10^{14} \mathrm{~W} / \mathrm{cm}^{2}$, well below $10^{16}$. Note moreover that $\mathrm{Xe}{ }^{10+}$ begins appearing with a population of $10^{-5}$ at an intensity of about $1.5 \times 10^{14} \mathrm{~W} / \mathrm{cm}^{2}$, while it never exceeds the populations of $\mathrm{Xe}^{7+}, \mathrm{Xe}^{8+}$ and $\mathrm{Xe}^{9+}$. Actually, the whole picture points rather clearly to the gradual leaking of the initial population through the sequence of ions. The peak values of the various ionic species, resulting from an interplay between the order of non-linearity and the value of the relevant cross section, reflect the rate of flow to and out of each ionic species. The abrupt changes of peak heights, as for example from $\mathrm{Xe}^{11+}$ to $\mathrm{Xe}^{12+}$, are due to changes of the order of non-linearity, as determined by the corresponding ionization potentials.

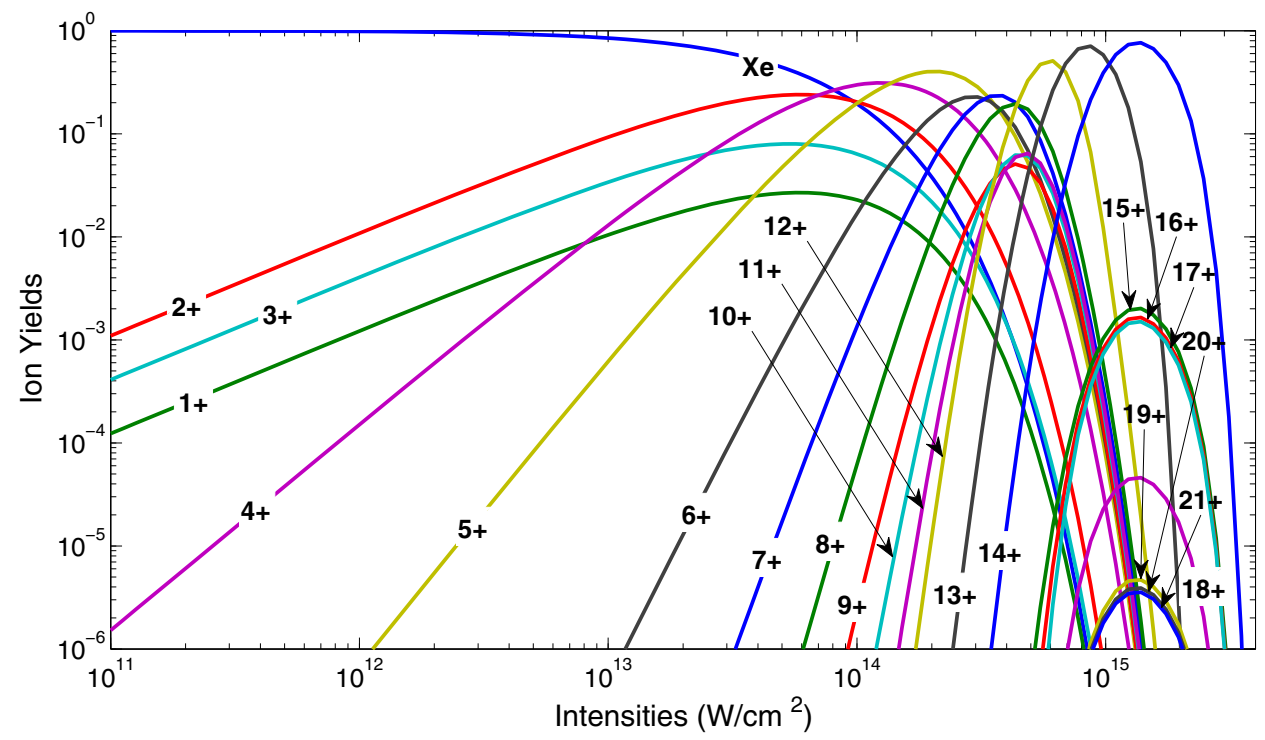

Figure 2. Calculated population of the neutral and generated ionic yields of Xe, as a function of FEL peak intensity, under $93 \mathrm{eV}$ radiation of $10 \mathrm{fs}$ pulse duration.

The overall picture then demonstrates that, if we were to postulate some unusual, even if unspecified, process that sets in at the higher intensities, say above $5 \times 10^{15}$ 
$\mathrm{W} / \mathrm{cm}^{2}$, leading to multiple electron ejection, it would have to originate from a species like $\mathrm{Xe}^{6+}$ and not from the neutral with its giant resonance. It should be clear by now that, ironically, the effect of the relatively high cross section around that "giant" resonance is to cause the rapid depletion of the neutral at lower intensities, precluding thus the possibility of its exposure to the higher peak intensity.

A few further remarks concerning certain details of the sequence of processes are perhaps in order here. The photo-ejection of one $4 \mathrm{~d}$ electron opens a hole whose filling by an Auger process, leads predominantly to the net ejection of 2 electrons, with (it bears repeating) known cross sections. That is why the dominant species up to intensities of about $5 \times 10^{13} \mathrm{~W} / \mathrm{cm}^{2}$ is $\mathrm{Xe}^{2+}$. The Auger lifetimes in this case are somewhat shorter than the pulse duration. Thus, in the scale of the pulse duration, the hole is in effect filled quickly, which means that the ejection of a $4 \mathrm{~d}$ electron from $\mathrm{Xe}^{2+}$ represents a significant channel, which is of course included in the calculation leading to the results of Fig. 2. It is true that $\mathrm{Xe}^{+}$and $\mathrm{Xe}^{2+}$ do exhibit a resonance at around that photon energy, but with values of the cross section significantly smaller than that of the neutral; which have anyway been incorporated in the rate equations. Beginning with $\mathrm{Xe}^{3+}$ and beyond, single-photon ionization is dominated by electron ejection from the $5 \mathrm{~s}$ and $5 \mathrm{p}$ shells. Be that as it may, all processes with significant cross sections, from all shells of importance, have been included in the rate equations. It is important to keep in mind that, in the experimental data which include yields from the peripheral volume of lower intensities, beyond the focal region, $\mathrm{Xe}^{2+}$ will always dominate as is actually the case in 2], Fig.4, as well as in our earlier work [5].

Theory can in addition provide a direct quantitative assessment of the evolution (depletion) of the neutral during the pulse. This is shown in Fig. 3, for pulses of various peak intensities, ranging from $10^{13}$ to $10^{16} \mathrm{~W} / \mathrm{cm}^{2}$, all of $10 \mathrm{fs}$ duration, confirming the picture discussed above in terms of the ionic yields. It is obvious, in particular, that for a peak intensity of $10^{16}$ or even $10^{15}$, the neutral has been depleted severely, long before the peak intensity is reached. Note, moreover, that although at $10^{14} \mathrm{~W} / \mathrm{cm}^{2}$ about $30 \%$ of the neutral is still available at the peak intensity (Fig. [3), the population of $\mathrm{Xe}^{10+}$ at that intensity is about 7 orders of magnitude lower.

Despite the apparent complexity of the problem, involving 23 differential equations and processes of non-linearity up to 7 , we came across a rather interesting surprise, 


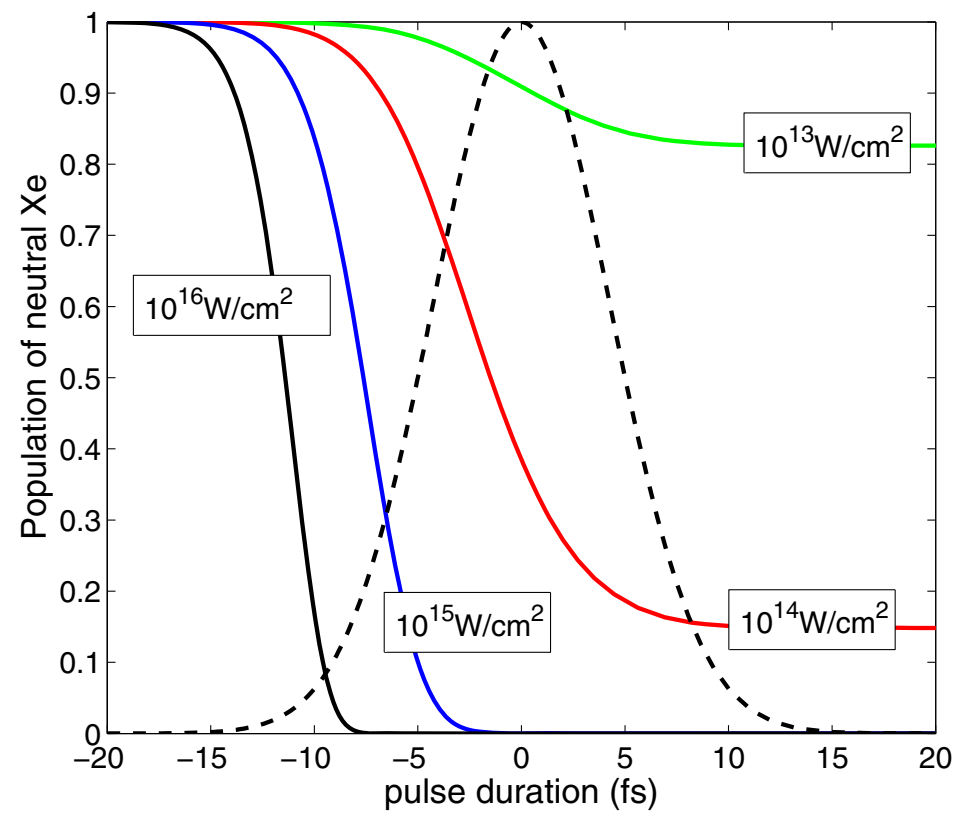

Figure 3. Evolution of the population of the neutral during the pulse for various peak intensities. The dashed line represents a pulse of 10 fs duration (FWHM).

contained in the results of Fig. 4 in comparison to those of Fig. 2, In Fig. 2, the yield of $\mathrm{Xe}^{3+}$, over a range of intensities spanning two orders of magnitude, is visibly lower than what it is in Fig. 4. The difference is due to the values of the single-photon partial cross section leading from $\mathrm{Xe}$ to $\mathrm{Xe}^{3+}$, employed in the two calculations (as indicated in the caption of Fig. (4). Although theoretical and experimental values do exist in the literature [6], a difference by a factor of 1.66 between the values employed in Fig. 2 and 廿4 is well within the known uncertainties. Surprisingly, or maybe not, the relative yields of $\mathrm{Xe}^{2+}$ and $\mathrm{Xe}^{3+}$ in Fig. 4 are in much better agreement with the experimental results of [2]. It appears therefore that despite the high intensity and many stages of ionization, the data (taken at face value) can provide a clue as to the most appropriate value of the cross section for $\mathrm{Xe} \rightarrow \mathrm{Xe}^{3+}$. Incidentally, this provides an additional redundancy check of the decisive role of single-photon transitions in the sequence of ionization events, as well as the validity of the rate equations.

\section{FURTHER EXPERIMENTAL CONFIRMATION OF LOPT}


Theory of Multiple Ionization of Xenon under strong XUV radiation and the role of the Giant Resonance.11

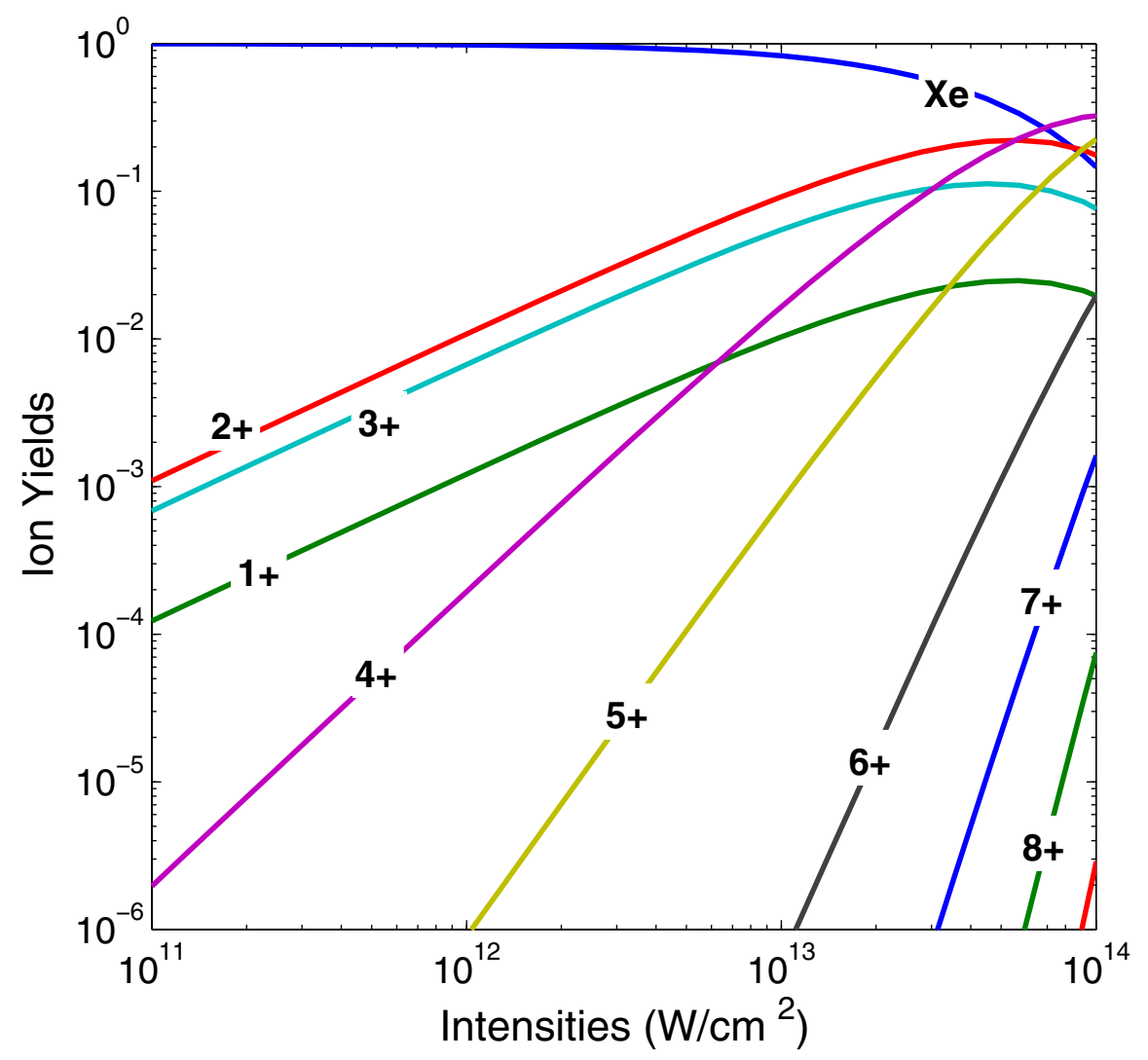

Figure 4. Sensitivity of the yields of $\mathrm{Xe}^{2+}$ and $\mathrm{Xe}^{3+}$ to the single-photon cross sections. The cross section for $\mathrm{Xe} \rightarrow \mathrm{Xe}^{3+}$ employed in the calculation for this Figure is $10 \mathrm{Mb}$ as compared to $6 \mathrm{Mb}$ in the calculation for Fig. 2

In case there was a lingering doubt as to the validity of LOPT under the conditions of these experiments, further confirmation has been provided by a more recent experimental paper [22], in which the additional absorption of one photon, above that exciting the giant resonance, has been documented through the analysis of the photoelectron energy spectrum. This is an ATI (Above Threshold Ionization) process, corresponding to a net 2-photon absorption from a $4 \mathrm{~d}$ electron. The slope of the respective photoelectron yield, as a function of FEL intensity (Fig.4, 22]) in a log-log plot, has been found to be equal to 2, an unmistakable signature of LOPT. As is well known from strong field non-perturbative behavior, the onset of deviation from LOPT is characterized by the departure of the slope of ATI from that corresponding to the number of absorbed photons (see, for example, [23]). On the basis of our results in Fig. 2 and 3 above, this is hardly surprising, since we have shown that the neutral is depleted at the lower intensities. As a consequence, any photo-absorption product originating directly from the neutral, as 
Theory of Multiple Ionization of Xenon under strong XUV radiation and the role of the Giant Resonance.12

the ATI electrons must be, will inevitably appear at the lower intensities, during the rise of the pulse, while the neutral is still around. Thus it goes without saying that any speculation about the behavior of Xe with its giant resonance at $10^{16} \mathrm{~W} / \mathrm{cm}^{2}$ is devoid of meaning in the context of these experiments. But even if the neutral were exposed to an intensity near $10^{16} \mathrm{~W} / \mathrm{cm}^{2}$ at this photon energy, as it might under a much shorter pulse, the behavior would still be within LOPT, as explained in our introduction above.

In the meantime, $\mathrm{Pi}$ and Starace 22 have published an ab initio value $5 \times 10^{-52}$ $\mathrm{cm}^{4} \mathrm{sec}$ for this ATI 2-photon (generalized) cross section at photon energy $93 \mathrm{eV}$, as obtained within LOPT. Using this cross section and that for single-photon absorption $(22 \mathrm{Mb})$, it is straightforward to calculate the ratio of the two-photon to the singlephoton rate, at a given intensity. For example, at an intensity of $10^{14} \mathrm{~W} / \mathrm{cm}^{2}$, that ratio turns out to be $2 \times 10^{-4}$, which means that the ATI yield is about four orders of magnitude smaller than the single-photon one, in reasonable agreement with what has been reported in 22]. On the other hand, if we were to use an intensity of $10^{16}$ $\mathrm{W} / \mathrm{cm}^{2}$, we would find this ratio to be $2 \times 10^{-2}$ or $2 \%$ which is in the ballpark of the theoretical estimate of $0.5 \%$ given in [22], but still quite different from the experimental value. Be that as it may, it should be clear by now that estimates for this ratio at such a high intensity are meaningless, because the neutral will never "see" an intensity of $10^{16} \mathrm{~W} / \mathrm{cm}^{2}$, for a $10 \mathrm{fs}$ pulse. It is a simple exercise to show that the lifetime of the neutral (against ionization) at that intensity is less than $10^{-16} \mathrm{sec}$.

\section{RELEVANCE OF COLLECTIVE PLASMA-LIKE OSCILLATIONS}

The above experimental data on ATI and their quantitative theoretical interpretation have direct bearing on a very recent attempt [25] at interpreting the multiple ionization of Xe in terms of an ad hoc semiclassical model, in which the eighteen 4d, 5s and 5p electrons are modeled by a one-dimensional, damped, harmonically driven oscillator. By representing ionization in terms of damping constants, and through an adjustment of parameters, a curve resembling the giant resonance has been obtained in Fig.2 of [25]. We shall refrain from commenting on the appropriateness and validity of such a model at this point in time, in view of the exhaustive information, accumulated 
over the last decades, on a well-established quantum phenomenon. But it is relevant and important to point out that any attempt at alternative theoretical models should at least be compatible with existing experimental data and of course self-consistent. There is, however, a serious difficulty in this case, because the model of [25] cannot possibly account for ATI which in view of 22] is an experimental fact, indisputably and quantitatively compatible with single-atom multiphoton theory. As we have shown above in detail, the behavior, magnitude and intensity dependence of that ATI yield prove, beyond any doubt, that the process begins with the ejection of one electron from the $4 \mathrm{~d}$ shell. The $4 \mathrm{~d}$ shell therefore begins breaking up very early in the pulse; precisely because of the large value of the cross section. One cannot have an ATI photoelectron without having an electron raised into the continuum by the absorption of one photon. And of course the observed energies of the photoelectrons correspond exactly to those dictated by energy conservation. It is evident therefore that the experimental data are incompatible with the assumption of the $4 \mathrm{~d}, 5 \mathrm{~s}$ and $5 \mathrm{p}$ shells oscillating at high intensity. Curiously, the experimental ATI paper [22] is not cited in 25], although its author is one of the co-authors of [22].

A few further remarks on this issue are in order at this point. (a) The fact that by adjusting parameters one can fit one feature of photoabsorption does not entail its validity, especially when it concerns a thoroughly studied and settled process, such as the giant resonance in Xenon. The above discussed ATI process is one of many others that cannot possibly be accommodated by the model of [25]. (b) The assertion that the model is not valid below $4 \times 10^{13} \mathrm{~W} / \mathrm{cm}^{2}$ poses another difficulty. As we have seen above, by the time this intensity is reached, the neutral has been depleted significantly. If we were to assume that the neutral remaining after that intensity, suddenly loses many electrons (of the order of 18), we would expect a sudden surge of ions around $\mathrm{Xe}^{18+}$. This however flies against the experimental data themselves (Fig.4, [2]), as well as our theory, because in both the ions are seen to follow a sequence of appearance. (c) The modeling in [25] is based on a sudden turn on of the coupling which is incompatible with the real pulse. And it is not an academic issue but a very practical one, because as it was demonstrated a long time ago [21] and reconfirmed here, the wings in the rise of the pulse play a decisive role on the depletion of species through the sequential channels. That is why, non-perturbative behavior at long (infrared) wavelengths came into play when 
Theory of Multiple Ionization of Xenon under strong XUV radiation and the role of the Giant Resonance.14

pulse durations broke the picosecond barrier. The corresponding barrier at XUV and beyond is of the order of sub-femtosecond. (d) The data moreover clearly demonstrate the sequential generation of successive ions according to well-known cross sections and the associated Auger processes, with $\mathrm{Xe}^{2+}$ dominating the onset of ionization, followed by $\mathrm{Xe}^{3+}$, as expected by LOPT. (e) Atoms are neither clusters nor plasmas. They have well known structure and their coupling to radiation obeys selection rules, no matter what the order of the photo-absorption process may be.

Finally, it may be helpful to remind the reader that ad hoc models for multiple ionization have been attempted long ago ([21] and references therein), with reasoning similar, but not identical, to that in [25]. Careful and detailed investigations, however, demonstrated that the claims were incompatible with the rules governing radiation-atom interactions, in relation to the pulse duration. That in fact was the motivation for the introduction of the scaling approach [21] which was instrumental in demonstrating the fallacy of the so-called collective multiple ionization. Ultimately, it was the examination of photoelectron energy spectra that settled the issue [26]. In the present case, the signature of the ATI photoelectron energy alone provides a valuable, in fact crucial, clue. Nevertheless, more detailed studies of photoelectron energy spectra, such as a recent example in $\mathrm{Kr}$ [27, will provide further insight as to the relative magnitude of the channels leading to multiple ionization.

\section{DISCUSSION AND OUTLOOK}

Through a quantitative analysis of the time evolution of the Xe ionic species under radiation of photon energy $93 \mathrm{eV}$, pulse duration $10 \mathrm{fs}$ and intensity up to $10^{16} \mathrm{~W} / \mathrm{cm}^{2}$, we have shown that neutral Xe is depleted well before that intensity is reached. As a result, the giant resonance with its large cross section cannot be a contributor to whatever may happen at intensities, say, above $5 \times 10^{14} \mathrm{~W} / \mathrm{cm}^{2}$. The fact that, with increasing peak intensity, significant yields of ionic species are produced within a larger part of the interaction volume (outside the focal region), does not affect the argument, because the intensity in the expanded volume is lower. Moreover, whatever approximations may be involved in the values of the higher order - 3-photon and above 
- cross sections, they do not affect the issue of the role of the giant resonance, since species up to and including $\mathrm{Xe}^{7+}$ are produced via single-photon sequential absorptions, with known cross sections, within LOPT. This has in fact been verified directly through a parametric study, in which we have varied the values of the higher order cross sections, within a reasonable range, with the result that it is only the populations of the higher ionic species that are redistributed somewhat, without affecting the overall picture (see Appendix). It should be further noted that the known intensity fluctuations of the FEL pulses [1, 20], do not affect our central argument. The yield of single-photon processes is not affected by the fluctuations, while their effect on N-photon processes is to increase the apparent N-photon cross section by at most a factor of N!, which again affects only the relative yields of the higher ionic species and not the neutral or the lower ionic species.

Still, in anticipation of future improvement of sources, there is a process of direct many electron ejection which is in principle possible and perhaps worth pondering. Given sufficient intensity, it is conceivable (technically speaking there is a Feynman diagram) that ten $4 \mathrm{~d}$ electrons can be ejected via an approximately 17 -photon process, for photon energies in the range of 100 to $200 \mathrm{eV}$, leading to the creation of a Xe atom with a hollow $4 \mathrm{~d}$ shell. This would be a process still within LOPT, but it would require a pulse duration well below $1 \mathrm{fs}$, so that the depletion of the neutral in the rise of the pulse can be avoided. The quantitative dynamics, lifetime and channels of decay of such a hollow state represent of course terra incognita and an enormously challenging problem. This idea of direct multiphoton multielectron processes, involving up to 8 electrons, has been discussed most recently [20] in a specific quantitative context. Thus if and when FEL sources can provide sub-fs, intense pulses, it will be worthwhile to explore in more detail the feasibility of the observation of such direct multielectron processes. Although the details of the underlying dynamics represent a daunting task, still multiphoton cross sections for such direct processes can be estimated through scaling [20]. It is then a matter of extending our rate equations, by including such higher non-linearity direct processes, and assessing their contribution to the laser power dependence of ionic species, as compared to that of the sequential alone, as discussed in [20]. Such a project must, however, await the development of sub-fs, intense pulses in this short wavelength range; because otherwise it may simply remain as an academic theoretical exercise. 
There is, however, one point that should be made clear at this point in time. The above direct, multielectron, multiphoton process has nothing to do with the giant resonance, which in any case is a single-photon process. All of the electrons in such direct processes are driven by the field, while in the giant resonance only one electron is ejected by the field, the role of correlation between the electrons in the shell being to lend enhanced oscillator strength to the single-photon transition. In contrast, in the direct process envisioned here, electron correlation would not be a major influence [20], since in a real sense each electron is pushed out by a photon. In fact, for a sufficiently short pulse, electron-electron interaction is completely negligible. Nevertheless, although in some sense the electrons would be ejected "together", instead of sequentially, we would refrain from using the term collective, as it may not be particularly helpful, if not misleading, since it conjures images of collective processes in heavy nuclei or mesoscopic systems which have hardly any resemblance to an atom.

\section{Acknowledgments}

The authors are grateful to George M. Nikolopoulos for helpful discussions and comments, to A. F. Starace for clarifying information related to the value of the ATI cross section and to Hugo van der Hart for supplementary information on his estimate of the ATI contribution in 22. Also detailed information on Xe cross section measurements by J. M. Bizau is gratefully acknowledged. Support by the European COST Action CM0702 is also acknowledged.

\section{Appendix A.}

The purpose of this Appendix is to provide the complete set of differential (rate) equations governing the production of the Xe ionic species by sequential multiphoton ionization, under radiation of photon energy $93 \mathrm{eV}$. The symbol $\mathrm{N}_{j}(\mathrm{j}=0,1,2,22)$ indicates the time-dependent population of the species of charge $\mathrm{j}+$, and $\dot{N}_{j}$ the time derivative.

The symbol $\sigma_{i j}^{(n)}$ denotes the single- or multiphoton cross section for the transition from ionic species $\mathrm{Xe}^{i+}$ to $\mathrm{Xe}^{j+}$, with the superscript $\mathrm{n}$ indicating the order of the process which appears also as the exponent of the time-dependent photon flux $\mathrm{F}(\mathrm{t})$. Absence of a superscript indicates a single-photon process. The calculation is limited to species up to and including $\mathrm{Xe}^{22+}$, because the highest species indicated in the experimental data in 3$]$ is $\mathrm{Xe}^{21+}$. Strictly speaking, there should also be terms in the right-hand 
Theory of Multiple Ionization of Xenon under strong XUV radiation and the role of the Giant Resonance.17

side of the equations representing the creation of the excited hole state and its decay, via the appropriate cascade. It turns out, however, that such terms do not affect the results appreciably, because the pulse duration is longer than the relevant lifetimes [6]. Such terms will be necessary when pulse durations shorter than the leading lifetimes become available. Since only data in the form of TOF peaks have been given in [3], a quantitative comparison with the laser power dependence shown in Fig. 2, as well as Fig. A1 below, is not possible. Qualitative comparison through visual inspection of the TOF spectra in [3] appears to show reasonable resemblance with theory.

The rate equations are:

$$
\begin{aligned}
& \dot{N}_{0}=-\left(\sigma_{01}+\sigma_{02}+\sigma_{03}\right) F N_{0} \\
& \dot{N}_{1}=\sigma_{01} F N_{0}-\sigma_{12} F N_{1}-\sigma_{13} F N_{1} \\
& \dot{N}_{2}=\sigma_{02} F N_{0}+\sigma_{12} F N_{1}-\sigma_{23} F N_{2}-\sigma_{24} F N_{2} \\
& \dot{N}_{3}=\sigma_{03} F N_{0}+\sigma_{13} F N_{1}+\sigma_{23} F N_{2}-\sigma_{34} F N_{3} \\
& \dot{N}_{4}=\sigma_{24} F N_{2}+\sigma_{34} F N_{3}-\sigma_{45} F N_{4} \\
& \dot{N}_{5}=\sigma_{45} F N_{4}-\sigma_{56} F N_{5}-\sigma_{56}^{(2)} F^{2} N_{5} \\
& \dot{N}_{6}=\sigma_{56} F N_{5}+\sigma_{56}^{(2)} F^{2} N_{5}-\sigma_{67} F N_{6}-\sigma_{67}^{(2)} F^{2} N_{6} \\
& \dot{N}_{7}=\sigma_{67} F N_{6}+\sigma_{67}^{(2)} F^{2} N_{6}-\sigma_{78}^{(2)} F^{2} N_{7} \\
& \dot{N}_{8}=\sigma_{78}^{(2)} F^{2} N_{7}-\sigma_{89}^{(2)} F^{2} N_{8} \\
& \dot{N}_{9}=\sigma_{89}^{(2)} F^{2} N_{8}-\sigma_{9,10}^{(2)} F^{2} N_{9}-\sigma_{9,10}^{(3)} F^{3} N_{9} \\
& \dot{N}_{10}=\sigma_{9,10}^{(2)} F^{2} N_{9}+\sigma_{9,10}^{(3)} F^{3} N_{9}-\sigma_{10,11}^{(2)} F^{2} N_{10}-\sigma_{10,11}^{(3)} F^{3} N_{10} \\
& \dot{N}_{11}=\sigma_{10,11}^{(2)} F^{2} N_{10}+\sigma_{10,11}^{(3)} F^{3} N_{10}-\sigma_{11,12}^{(2)} F^{2} N_{11}-\sigma_{11,12}^{(3)} F^{3} N_{11} \\
& \dot{N}_{12}=\sigma_{11,12}^{(2)} F^{2} N_{11}+\sigma_{11,12}^{(3)} F^{3} N_{11}-\sigma_{12,13}^{(4)} F^{4} N_{12} \\
& \dot{N}_{13}=\sigma_{12,13}^{(4)} F^{4} N_{12}-\sigma_{13,14}^{(4)} F^{4} N_{13} \\
& \dot{N}_{14}=\sigma_{13,14}^{(4)} F^{4} N_{13}-\sigma_{14,15}^{(4)} F^{4} N_{14} \\
& \dot{N}_{15}=\sigma_{14,15}^{(4)} F^{4} N_{14}-\sigma_{15,16}^{(5)} F^{5} N_{15}
\end{aligned}
$$


Theory of Multiple Ionization of Xenon under strong XUV radiation and the role of the Giant Resonance.18

$$
\begin{aligned}
& \dot{N}_{16}=\sigma_{15,16}^{(5)} F^{5} N_{15}-\sigma_{16,17}^{(5)} F^{5} N_{16} \\
& \dot{N}_{17}=\sigma_{16,17}^{(5)} F^{5} N_{16}-\sigma_{17,18}^{(5)} F^{5} N_{17} \\
& \dot{N}_{18}=\sigma_{17,18}^{(5)} F^{5} N_{17}-\sigma_{18,19}^{(6)} F^{6} N_{18} \\
& \dot{N}_{19}=\sigma_{18,19}^{(6)} F^{6} N_{18}-\sigma_{19,20}^{(7)} F^{7} N_{19} \\
& \dot{N}_{20}=\sigma_{19,20}^{(7)} F^{7} N_{19}-\sigma_{20,21}^{(7)} F^{7} N_{20} \\
& \dot{N}_{21}=\sigma_{20,21}^{(7)} F^{7} N_{20}-\sigma_{21,22}^{(7)} F^{7} N_{21} \\
& \dot{N}_{22}=\sigma_{21,22}^{(7)} F^{7} N_{21}
\end{aligned}
$$

while the values of the cross sections, in units $\mathrm{cm}^{2 N} \mathrm{sec}^{N-1}$ for an N-photon process, employed in the calculation whose results are shown in Fig. 2 are:

$$
\begin{aligned}
& \sigma_{01}=1.8 \cdot 10^{-18} \quad \sigma_{02}=16 \cdot 10^{-18} \quad \sigma_{03}=6 \cdot 10^{-18} \\
& \sigma_{12}=2 \cdot 10^{-18} \quad \sigma_{13}=23.5 \cdot 10^{-18} \quad \sigma_{23}=3 \cdot 10^{-18} \\
& \sigma_{24}=22.5 \cdot 10^{-18} \quad \sigma_{34}=48 \cdot 10^{-18} \quad \sigma_{45}=20 \cdot 10^{-18} \\
& \sigma_{56}=5 \cdot 10^{-20} \quad \sigma_{56}^{(2)}=10^{-48} \quad \sigma_{67}=5 \cdot 10^{-20} \quad \sigma_{67}^{(2)}=10^{-48} \\
& \sigma_{78}^{(2)}=7 \cdot 10^{-49} \quad \sigma_{89}^{(2)}=7 \cdot 10^{-49} \quad \sigma_{9,10}^{(2)}=10^{-49} \quad \sigma_{9,10}^{(3)}=2 \cdot 10^{-79} \\
& \sigma_{10,11}^{(2)}=8 \cdot 10^{-50} \quad \sigma_{10,11}^{(3)}=10^{-79} \quad \sigma_{11,12}^{(2)}=6 \cdot 10^{-50} \quad \sigma_{11,12}^{(3)}=8 \cdot 10^{-80} \\
& \sigma_{12,13}^{(4)}=10^{-112} \quad \sigma_{13,14}^{(4)}=10^{-113} \quad \sigma_{14,15}^{(4)}=10^{-114} \quad \sigma_{15,16}^{(5)}=10^{-143} \\
& \sigma_{16,17}^{(5)}=10^{-143} \quad \sigma_{17,18}^{(5)}=10^{-143} \quad \sigma_{18,19}^{(6)}=10^{-172.8} \\
& \sigma_{19,20}^{(7)}=10^{-203} \quad \sigma_{20,21}^{(7)}=10^{-203} \quad \sigma_{21,22}^{(7)}=10^{-203}
\end{aligned}
$$

For single-photon processes (up to $\mathrm{Xe}^{7+}$ ), the cross sections have been taken from [6, 8, 9], as well as checked through independent calculation using Cowan's code (http://aphysics2.lanl.gov/cgi-bin/ION/runlanl08d.pl ). Multiphoton cross sections have been obtained mainly through scaling [21], while some 2-photon cross sections have also been calculated through a truncated summation.

It may be useful to devote here some space on the level of accuracy of the multiphoton cross sections, as obtained through scaling. First recall that a single-photon cross section is proportional to the square of a dipole matrix element between the initial and 
Theory of Multiple Ionization of Xenon under strong XUV radiation and the role of the Giant Resonance.19 final state. An N-photon transition amplitude involves a summation over (N-1) manifolds of intermediate states, in which a product of $\mathrm{N}$ dipole matrix elements appears in the numerator [21. As usual, the cross section is given by the square of the absolute value of the transition amplitude. Dimensionally, therefore, it involves a product of $\mathrm{N}$ squared matrix elements. Now, an error of a factor of 2 in the squared single-photon matrix element, entails an error of a factor of 2 in the single-photon cross section. It follows then that, an error of a factor of 100 in the value of, say, a 6-photon cross section, is equivalent to an error of $100^{1 / 6}=2.16$, essentially the same as a factor of 2 in the single-photon case. As explained in detail in [21], the scaling procedure is exact for hydrogen-like systems. Its systematic extension to other atoms takes into account the size of the atom, as well as the ionization potential, both of which reflect the particular atomic structure of the species under consideration. Thus although no longer exact, through numerous applications and testing over the years, it has been found to be well within the accuracy of the relevant experiments in which the only measured quantity is the laser power dependence of ionic yields.

The orders of the various ionization processes are summarized below

$$
\begin{aligned}
& X e \rightarrow(1+),(2+),(3+): 1 p h \quad(1+) \rightarrow(2+),(3+): 1 p h \quad(2+) \rightarrow(3+),(4+): 1 p h \\
& (3+) \rightarrow(4+): 1 p h \quad(4+) \rightarrow(5+): 1 p h \quad(5+) \rightarrow(6+): 1 p h, 2 p h \\
& (6+) \rightarrow(7+): 1 p h, 2 p h \quad(7+) \rightarrow(8+): 2 p h \quad(8+) \rightarrow(9+): 2 p h \\
& (9+) \rightarrow(10+): 2 p h, 3 p h \quad(10+) \rightarrow(11+): 2 p h, 3 p h \quad(11+) \rightarrow(12+): 2 p h, 3 p h \\
& (12+) \rightarrow(13+): 4 p h \quad(13+) \rightarrow(14+): 4 p h \quad(14+) \rightarrow(15+): 4 p h \\
& (15+) \rightarrow(16+): 5 p h \quad(16+) \rightarrow(17+): 5 p h \quad(17+) \rightarrow(18+): 5 p h \\
& (18+) \rightarrow(19+): 6 p h \quad(19+) \rightarrow(20+): 7 p h \\
& (20+) \rightarrow(21+): 7 p h \quad(21+) \rightarrow(22+): 7 p h
\end{aligned}
$$

In order to give the reader a sense of the sensitivity of the results to the cross sections, we present below ionic yields for a set of modified, but still within a reasonable range of values, multiphoton cross sections. As already mentioned in the main text, the only change consists of a slight redistribution of mostly the populations of the higher 
Theory of Multiple Ionization of Xenon under strong XUV radiation and the role of the Giant Resonance.20 species.

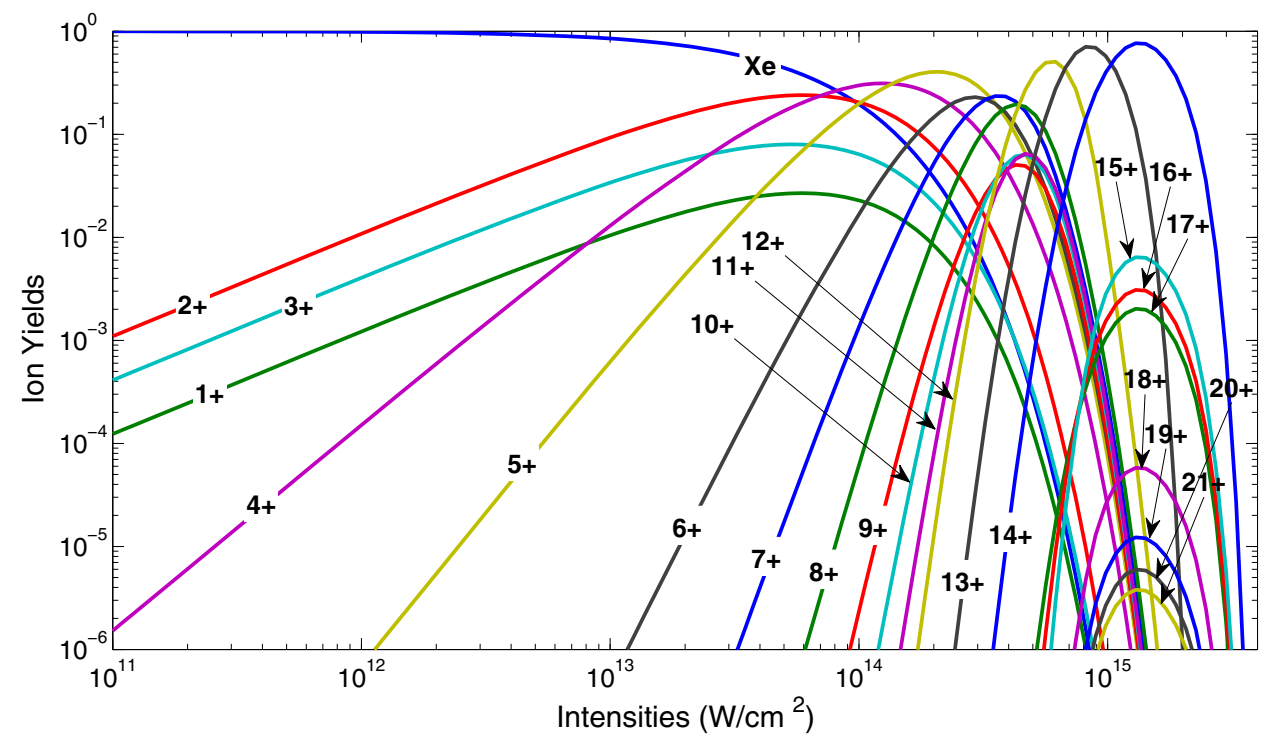

Figure A1. Calculated populations of the generated ionic species of Xe with the following alternative set of values for the higher order cross sections: $\sigma_{16,17}=5 \cdot 10^{-144}$, $\sigma_{17,18}^{(5)}=2 \cdot 10^{-144}, \sigma_{18,19}^{(6)}=10^{-173}, \sigma_{19,20}^{(7)}=10^{-203} \mathrm{~cm}^{14} \cdot \sec ^{6}, \sigma_{20,21}^{(7)}=5 \cdot 10^{-204}$, $\sigma_{21,22}^{(7)}=2 \cdot 10^{-204}$.

\section{References}

[1] T.E. Glover et al Nature Physics 669 (2010).

[2] A.A. Sorokin et al Phys. Rev. Lett. 99213002 (2007).

[3] M. Richter et al Phys. Rev. Lett. 102163002 (2009).

[4] M. Richter et al J. Phys. B: At. Mol. Opt. Phys. 43194005 (2010) and references therein.

[5] M.G. Makris et al Phys. Rev. Lett. 102033002 (2009).

[6] U. Becker, D. Szostak, H.G. Kerkhoff, M. Kupsch, B. Langer, R. Wehlitz, A. Yagishita and T. Hayaishi Phys. Rev. A 393902 (1989); also F. Penent et al Phys. Rev. Lett. 95083002 (2005).

[7] A.F. Starace, in "Handbook der Physik", edited by W. Melhorn (Springer Verlag, Berlin 1982, Vol 31, p. 1.

[8] P. Andersen et al J. Phys. B: At. Mol. Opt. Phys. 342009 (2001).

[9] J.M. Bizau et al Phys. Rev. A 73022718 (2006).

[10] K.T. Cheng and C.Froese Fischer, Phys. Rev. A 282811 (1983).

[11] K.T. Cheng and W.R. Johnson, Phys. Rev. A 282820 (1983).

[12] D.J. Kennedy and S.T. Manson, Phys. Rev. A 5227 (1972).

[13] W.R. Johnson and K. T. Cheng, Phys. Rev. A 20978 (1979). 
Theory of Multiple Ionization of Xenon under strong XUV radiation and the role of the Giant Resonance.21

[14] A. Zangwill and P. Soven, Phys. Rev. A 211561 (1980).

[15] D. Toffoli, M. Stener and P. Decleva J. Phys. B: At. Mol. Opt. Phys. 351275 (2002).

[16] M.A. Kornberg and P. Lambropoulos J. Phys. B 32 L603 (1999).

[17] J.Feist et al Phys. Rev. A 77043420 (2008).

[18] E. Foumouo et al J. Phys. B 41051001 (2008).

[19] H. Bachau, Phys. Rev. A 83033403 (2011).

[20] P. Lambropoulos et al Phys. Rev. A 83021407 (2011).

[21] P. Lambropoulos and X. Tang, J. Opt. Soc. Am. B 4821 (1987); A.L. Robinson, Science 2321193 (1986); P. Lambropoulos, Comments At. Mol. Phys. 20199 (1987).

[22] V. Richardson et al Phys. Rev. Lett. 105013001 (2010).

[23] E. Cormier and P. Lambropoulos, J. Phys. B: At. Mol. Opt. Phys. 3077 (1997).

[24] L.W. Pi and A.F. Starace, Phys. Rev. A 82053414 (2010).

[25] M. Richter, J. Phys. B: At. Mol. Opt. Phys. 44075601 (2011).

[26] A.L. Robinson, Science 2321993 (1986).

[27] M. Meyer et al Phys. Rev. Lett. 104213001 (2010). 